\title{
The impact of pharmacy mediated conversion from intravenous to oral corticosteroids in patients with an exacerbation of chronic obstructive pulmonary disease
}

\author{
Mohannad Alshibani ${ }^{1,2}$, Ahmed Basilim ${ }^{1,2}$, Terri Warholak ${ }^{1}$ and Stephen J Perona ${ }^{3 *}$ \\ ${ }^{1}$ The University of Arizona, College of Pharmacy, Tucson, AZ, USA \\ ${ }^{2}$ Faculty of Pharmacy, King Abdulaziz University, Jeddah, Saudi Arabia \\ ${ }^{3}$ Department of Pharmacy, Northwest Medical Center, Tucson, AZ, USA
}

\begin{abstract}
Purpose: The purpose of this study was to evaluate a pharmacist initiated conversion program from intravenous (IV) to oral corticosteroids in patients with chronic obstructive pulmonary disease (COPD) exacerbations.

Methods: We conducted a retrospective observational study of patients who were admitted with a diagnosis of COPD exacerbation. The historical control group, included patients admitted between October 1 and December 31, 2013, did not have any intervention from pharmacists regarding conversion. The post intervention group, included patients admitted between October 1 and December 31, 2014, exposed to the new conversion program. Electronic medical records were reviewed for: time needed for conversion, hospital length of stay, and readmission rate for both groups.
\end{abstract}

Results: There were a total of 122 patients in the study. A low number of patients in the post intervention group ( $\mathrm{n}=11,22 \%)$ received pharmacist intervention. No significant difference was observed in hospital length of stay, time to conversion, or 30-day readmission rates. More patients in the intervention group received methylprednisolone as initial IV therapy $(P=0.002)$.

Conclusions: Among patients with COPD exacerbations, the automatic pharmacist medicated conversion program did not affect time to conversion, hospital length of stay, or readmission rates.

\section{Introduction}

\section{Background}

Chronic obstructive pulmonary disease (COPD) is a common respiratory condition involving the airways characterized by airflow limitation [1,2]. It affects almost 15.7 million American (6.4\%) and is associated with high morbidity and mortality [3]. It was considering the third-ranked cause of death in the United States in 2014 [4]. As a consequence of its high prevalence and chronicity, COPD causes high resource utilization with frequent clinician office visits, frequent hospitalizations due to acute exacerbations, and the need for chronic therapy.

There are numerous published reports demonstrating the benefit of conversion from intravenous (IV) to oral therapy including antimicrobials, acid suppressants, and antihypertensives [5-7]. This creates a mutually beneficial relationship for the hospital and patient by decreasing drug costs, reducing the risks of adverse drug reactions related to IV access, and potentially shortening hospital length of stay [5-7].

\section{Importance}

The current Global Initiative for Chronic Obstructive Lung Disease (GOLD) guidelines recommend the use of oral prednisone $40 \mathrm{mg}$ per day for 5 days for acute exacerbations of COPD [8]. The benefits of using systemic corticosteroids are to shorten recovery time, improve lung function and arterial hypoxemia, and reduce the risk of early relapse [8]. According to a large Cochrane review, there was no difference between parenteral and oral corticosteroids in treatment failure, relapse, or mortality [9]. Based on current literature, the use of oral corticosteroids in patients with COPD exacerbations is as effective as IV administration, and would be preferred in cases where patients can safely absorb oral medications. Low dose oral corticosteroids are not associated with worse outcomes than high dose IV treatment, and the short course treatment of systemic corticosteroids is not inferior to long course treatment [10-13]. Thus, the treatment with oral corticosteroids for a short period of time seems to be a reasonable approach in treating patients with an acute exacerbation of COPD.

The pharmacist can play a significant part in conversion of IV to oral medications once it is clinically appropriate. Several studies have shown the appropriate conversion from IV to oral antibiotics can decrease the length of hospitalization, reduce hospital cost, and improve patient care when this is accompanied with pharmacist intervention to evaluate patients' eligibility to switch [7]. To the best of

*Correspondence to: Stephen J. Perona, Department of Pharmacy, Northwest Medical Center ,6200 N La Cholla Blvd, Tucson, AZ 85741, Tel: 520-469-8692; Fax: 520-469-8695; E-mail: stephen.perona@northwestmedicalcenter.com

Key words: chronic obstructive pulmonary disease (COPD) exacerbations, systemic corticosteroids, intravenous to oral conversion, pharmacist intervention

Received: May 06, 2017; Accepted: June 09, 2017; Published: June 13, 2017 
Alshibani M (2017) The impact of pharmacy mediated conversion from intravenous to oral corticosteroids in patients with an exacerbation of chronic obstructive pulmonary disease

our knowledge a pharmacist mediated automatic conversion program for corticosteroids has not been previously described.

In September 2014, the local Pharmacy and Therapeutics Committee approved a new conversion program allowing pharmacists to convert from parenteral to oral corticosteroids in eligible patients. Inclusion criteria: (1) functioning GI tract defined as tolerance of an oral or liquid diet or oral medications, (2) 24 or more hours since admission, and (3) diagnosis of COPD exacerbation. Exclusion criteria: (1) severe nausea/vomiting or any other condition that compromises absorption of oral therapy, (2) requirement for mechanical ventilation or bilevel positive airway pressure (BiPAP), (3) prescriber indicates "Do not substitute route" in order, and (4) conversion to IV after initial PO therapy. Per hospital policy, all IV corticosteroids were converted back to oral prednisone $40 \mathrm{mg}$ daily for eligible patients.

\section{Goal of this investigation}

The primary objective of this study was to evaluate a newly approved conversion program allowing pharmacist mediated conversion from IV to oral corticosteroids in patients with COPD exacerbations.

\section{Methods}

\section{Study design}

This was a retrospective observational study conducted between December 2014 and May 2015. The study was conducted in a 300-bed community medical center, with an estimated annual census of 76,000 patients. Institutional review board approval was obtained prior to conducting the study.

\section{Selection of participants}

The study used a convenience sample of patients admitted to our facility. A pharmacist investigator used a computerized medical record system to identify patients discharged with an International Classification of Disease, Ninth Revision (ICD-9) code of 491, 492, or 496 indicating a diagnosis of COPD exacerbation. These three codes were selected to match the COPD readmission rates calculated by the Center for Medicare \& Medicaid Services.

We used a historical control group consisting of patients admitted between October 1 and December 31, 2013, prior to conversion program implementation. A post intervention group consisted of patients admitted between October 1 and December 31, 2014, after the implementation of the new conversion program. Patients were eligible if they met the following criteria: (1) were aged 18 years or older, and (2) had confirmed COPD exacerbation diagnosis. Patients were excluded if they had asthma.

\section{Data collection and processing}

Outcomes: Data collection included patient demographic data and home medications relevant to COPD treatment, number of days of IV and oral corticosteroid therapy, time to conversion eligibility, time to conversion to oral corticosteroids, length of hospital stay, and readmission within 30 days due to COPD exacerbations. The primary outcome was to evaluate time to conversion from IV to oral. The secondary outcomes were to identify hospital length of stay and 30-day readmission rates due to COPD exacerbation.

Data analysis: Hospital length of stay and time to conversion were evaluated using a student's t-test. The secondary outcome of hospital readmission rate within 30 days was evaluated using the chi square test. Demographic characteristics including categorical variables were assessed using a chi-square analysis unless the sample size for a case was less than 5, and in those cases, a Fisher's exact test was used. The unpaired Student's t test was used to compare all normally distributed continuous variables. The unpaired Student's t test was also used to compare differences in days until a conversion criterion was met, the mean day of conversion, the number of days of IV and oral corticosteroids. We calculated 50 patients in each group would provide $80 \%$ power to detect a 1.25 -day difference in time to conversion. We estimated 253 patients in each group would be needed to detect a 0.9 -day difference in hospital length of stay. The a-priori level of significance used for all analyses was $P<0.05$.

\section{Results}

\section{Characteristics of study subjects}

A total of 122 patients were identified using ICD-9 codes. After exclusion of 10 patients, a total of 112 patients were included in the analysis. All of the excluded patients were from the historical control group (Figure 1). Overall, patients were found to be elderly with a low incidence of comorbid conditions such as pneumonia and congestive heart failure that did not differ between groups $(14.8 \%$ vs $23.5 \%$; $P=$ $0.33)$ and $(8.2 \%$ vs $13.7 \% ; P=0.38)$ respectively. The overall severity of exacerbation as measured by intensive care unit ICU admission, use of BiPAP, or use of mechanical ventilation was low and similar between the two groups (see Table 1).

The mean duration of IV corticosteroid use did not differ between the control and post intervention groups $(2.9 \pm 1.7$ days vs $2.8 \pm 1.9$ days; $P=0.62$ ). There was no difference in the mean duration of oral corticosteroids $(1.3 \pm 1.8$ days vs $1.4 \pm 1.9$ days; $P=0.82)$. More patients in the post intervention group received methylprednisolone as initial IV therapy (42 patients [82.4\%]) vs 33 patients [54.1\%]; $P$ $=0.002$ ). However, there was no difference between the two groups when we calculated the total daily methylprednisolone equivalent dose (167 mg vs $179 \mathrm{mg} ; P=0.55$ ) (Table 1$)$.

\section{Main results}

The main results in this project showed that pharmacist intervention to switch from IV to oral corticosteroids was done only on 11 patients [22\%] in the intervention group. The median length of stay was similar between the control and the post intervention groups ( $4.4 \pm 2.3$ days vs $4.8 \pm 2.9$ days; $P=0.09$ ). Also, there was no significance difference between the two groups in the mean time to conversion

$(2.2 \pm 1.8$ days vs $2.8 \pm 2.2$ days; $p=0.35)$. The mean time to conversion eligibility was similar ( $1.2 \pm 0.7$ days vs $1.52 \pm 1.1$ days; $P=0.50)$. There was no significant difference in 30-day readmission rates (15 patients [24.6\%] vs 17 patients [33.3\%]; $P=0.40$ ), or 30 -day readmission rates due to COPD exacerbations (7 patients [11.5\%] vs 10 patients [19.6\%]; $P=0.34$ ) (Table 2 ).

\section{Discussion}

A key finding of this analysis is that the automatic conversion program was underutilized. The conversion rate of $22 \%$ is lower than expected, and there was a one-day difference between eligibility and actual conversion implying that pharmacist did not convert patients who were eligible. Although the study was not designed to evaluate the adherence to the conversion protocol or reasons for IV to oral conversion we believe the low intervention rate could be the result of several factors. There may be a perception of reduced efficacy switching 


\section{Total number of patients} $(\mathrm{n}=122)$

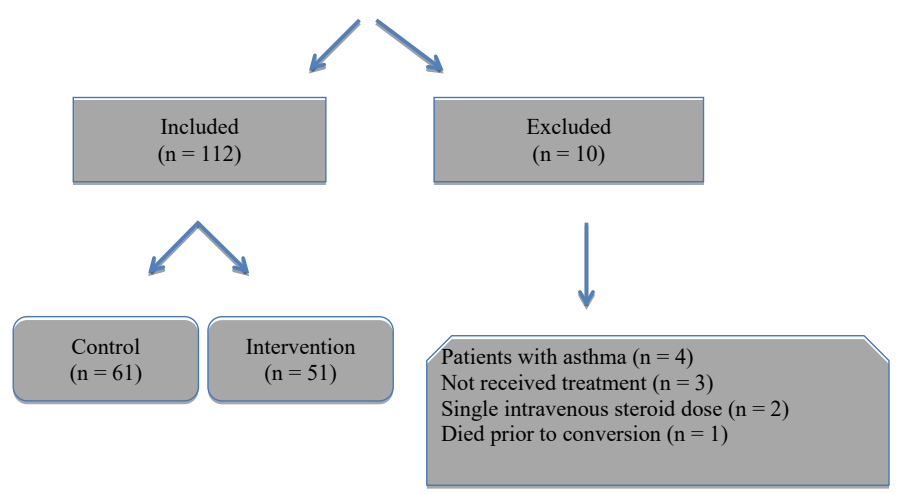

Figure 1. Flow of patients through the study

Table 1. Baseline Characteristics

\begin{tabular}{|c|c|c|c|}
\hline Variable & $\begin{array}{c}\text { Control } \\
(\mathbf{n}=\mathbf{6 1})\end{array}$ & $\begin{array}{c}\text { Intervention } \\
(\mathbf{n}=\mathbf{5 1})\end{array}$ & $\boldsymbol{P}$-value \\
\hline Age, years (Mean, SD) & $71(9.9)$ & $71(12.6)$ & 0.14 \\
\hline Male (n, \%) & $25(40.9)$ & $23(45.1)$ & 0.70 \\
\hline Pneumonia (n, \%) & $9(14.8)$ & $12(23.5)$ & 0.33 \\
\hline CHF (n, \%) & $5(8.2)$ & $7(13.7)$ & 0.38 \\
\hline ICU admission (n, \%) & $5(8.2)$ & $4(7.8)$ & 1.00 \\
\hline BiPAP use (n, \%) & $5(8.2)$ & $6(11.8)$ & 0.54 \\
\hline Ventilator use (n, \%) & $2(3.3)$ & $2(3.9)$ & 1.00 \\
\hline Duration of IV corticosteroids, days (Mean, SD) & $2.9(1.7)$ & $2.8(1.9)$ & 0.62 \\
\hline Duration of oral corticosteroids, days (Mean, SD) & $1.3(1.8)$ & $1.4(1.9)$ & 0.82 \\
\hline IV methylprednsisolone use (n, \%) & $33(54.1)$ & $42(82.4)$ & 0.002 \\
\hline IV methylprednsisolone equivalent dose, mg/ & $167(78.6)$ & $179(80.3)$ & 0.55 \\
\hline day (Mean, SD) & & & \\
\hline
\end{tabular}

${ }^{\mathrm{a}} \mathrm{CHF}=$ congestive heart failure, $\mathrm{ICU}=$ intensive care $\mathrm{unit}, \mathrm{BiPAP}=$ bilevel positive airway pressure, $\mathrm{IV}=$ intravenous, $\mathrm{n}=$ number, $\mathrm{SD}=$ standard deviation.

Table 2. Main Outcomes ${ }^{\mathrm{a}}$

\begin{tabular}{|c|c|c|c|}
\hline Variable & $\begin{array}{c}\text { Control } \\
(\mathbf{n = 6 1 )}\end{array}$ & $\begin{array}{c}\text { Intervention } \\
(\mathbf{n = 5 1 )}\end{array}$ & $\boldsymbol{P}$-value \\
\hline Length of stay, days (Mean, SD) & $4.4(2.3)$ & $4.8(2.9)$ & 0.09 \\
\hline Time to conversion, days (Mean, SD) & $2.2(1.8)$ & $2.8(2.2)$ & 0.70 \\
\hline Time to conversion eligibility, days (Mean, SD) & $1.2(0.7)$ & $1.5(1.1)$ & 0.33 \\
\hline 30-day readmission rates (n, \%) & $15(24.6)$ & $17(33.3)$ & 0.40 \\
\hline 30-day readmission rates due to COPD (n, \%) & $7(11.5)$ & $10(19.6)$ & 0.34 \\
\hline
\end{tabular}

${ }^{\mathrm{a}} \mathrm{COPD}=$ chronic obstructive pulmonary disease, $\mathrm{n}=$ number, $\mathrm{SD}=$ standard deviation.

from high dose IV to moderate dose oral corticosteroids. Other clinical activities such as therapeutic dosing or renal dose adjustment may take priority over IV to oral interchange. Although no cases were identified during our study period, some pharmacists cited that some physicians requested for "Do not substitute route" as a reason for not participating in conversion. In addition, data collection for the intervention group occurred immediately following program implementation, and it is possible the rate of intervention will increase over time as staff become accustomed to a new clinical activity. All of these factors could contribute to the low intervention rate observed in our study.

Several important details regarding the treatment of COPD exacerbations at our facility emerged during the study period. The average daily dose and duration of IV corticosteroids observed, approximately $170 \mathrm{mg}$ methylprednisolone for almost 3 days, is inconsistent with the current treatment recommendations for moderate dose oral corticosteroids [8,10-13]. Moreover, it was noted that our facility favors the use of IV methylprednisolone over dexamethasone as it was shown in the post intervention group compared with control group. An existing policy allows pharmacists to interchange methylprednisolone and dexamethasone in time of drug shortages possibly accounting for the lower methylprednisolone utilization in the control group [14]

Nevertheless, the use of oral prednisone should be encouraged in patients with acute exacerbations of COPD consistent with current evidence and guidelines recommendations [8-12]. As a result of this analysis, local treatment practices involving high dose IV corticosteroids as a standard initial therapy should be reevaluated. Further investigation into the rationale for prescribing high dose IV corticosteroids as initial therapy and delaying conversion to oral could support the development of targeted educational initiatives or inclusion of prompts in the Electronic Health Record to encourage appropriate prescribing practices. The use of pharmacy trainees, such as students or residents, to review and convert patient on a daily basis could improve adherence to the conversion protocol. Additionally, this intervention could be utilized to preserve the supply of IV corticosteroids in time of drug shortages.

This study has several limitations. First, the results cannot be generalized to all patients with COPD exacerbations since we excluded patients with asthma or who were on BiPAP or ventilation from conversion. Second, the use of diagnostic codes to identify patients might not capture all eligible patients. Third, our study had reduced study power for the following reasons: (1) a low percentage of patients received the intervention, (2) data collection for the intervention group occurred immediately following program initiation, and (3) our study was not powered to assess hospital length of stay.

In conclusion, an automatic pharmacist mediated conversion program did not affect time to conversion, hospital length of stay, or readmission rates. However, we believe the use of oral corticosteroids should be encouraged in patients with COPD exacerbations. During a shortage of parenteral corticosteroids this intervention could be utilized to preserve supply.

\section{References}

1. Buist AS, McBurnie MA, Vollmer WM, Gillespie S, Burney P, et al. (2007) International variation in the prevalence of COPD (the BOLD Study): a populationbased prevalence study. Lancet 370: 741-750. [Crossref]

2. Gershon AS, Warner L, Cascagnette P, Victor JC, To T (2011) Lifetime risk of developing chronic obstructive pulmonary disease: a longitudinal population study. Lancet 378: 991-996. [Crossref]

3. Wheaton AG, Cunningham TJ, Ford ES, Croft JB; Centers for Disease Control and Prevention (CDC) (2015) Employment and activity limitations among adults with chronic obstructive pulmonary disease-United States, 2013. MMWR 64: 290-295. [Crossref]

4. http://www.cdc.gov/nchs/hus. Accessed June 20, 2016.

5. Dasta JF, Boucher BA, Brophy GM, Cohen H, Hassan E, et al (2010) Intravenous to oral conversion of antihypertensives: a toolkit for guideline development. Ann Pharmacother 44: 1430-1447. [Crossref]

6. Ramirez JA, Vargas S, Ritter GW, Brier ME, Wright A, et al. (1999) Early switch from intravenous to oral antibiotics and early hospital discharge: a prospective observational study of 200 consecutive patients with community-acquired pneumonia. Arch Intern Med 159: 2449-2454. [Crossref]

7. Kuti JL, Le TN, Nightingale CH, Nicolau DP, Quintiliani R (2002) Pharmacoeconomics of a pharmacist-managed program for automatically converting levofloxacin route from i.v. to oral. AJHP 59: 2209-2215.

8. http://goldcopd.org/global-strategy-diagnosis-management-prevention-copd-2016 Accessed March 17, 2016. 
Alshibani M (2017) The impact of pharmacy mediated conversion from intravenous to oral corticosteroids in patients with an exacerbation of chronic obstructive pulmonary disease

9. Walters JA, Tan DJ, White CJ, Gibson PG, Wood-Baker R, et al. (2014) Systemic corticosteroids for acute exacerbations of chronic obstructive pulmonary disease. Cochrane Database Syst Rev 9: CD001288.

10. de Jong YP, Uil SM, Grotjohan HP, Postma DS, Kerstjens HA, et al. (2007) Oral or IV prednisolone in the treatment of COPD exacerbations: a randomized, controlled, double-blind study. Chest 132: 1741-1747. [Crossref]

11. Leuppi JD, Schuetz P, Bingisser R, Bodmer M, Briel M, et al. (2013) Short-term vs conventional glucocorticoid therapy in acute exacerbations of chronic obstructive pulmonary disease: the REDUCE randomized clinical trial. JAMA 309: 2223-2231.
12. Al-Habet S, Rogers HJ (1980) Pharmacokinetics of intravenous and oral prednisolone. Br J Clin Pharmacol 10: 503-508. [Crossref]

13. Lindenauer PK, Pekow PS, Lahti MC, Lee Y, Benjamin EM, et al. (2010) Association of corticosteroid dose and route of administration with risk of treatment failure in acute exacerbation of chronic obstructive pulmonary disease. JAMA 303: 2359-2367. [Crossref]

14. http://www.accessdata.fda.gov/scripts/drugshortages/. Accessed June 15, 2016.

Copyright: (2017 Alshibani M. This is an open-access article distributed under the terms of the Creative Commons Attribution License, which permits unrestricted use, distribution, and reproduction in any medium, provided the original author and source are credited. 